\title{
RÉCEPTION ET TRANSFORMATION DES IDÉES GÉOGRAPHIQUES DE L'ÉCOLE FRANÇAISE DE GÉOGRAPHIE AU JAPON
}

\author{
HIDEKI NOZAWA ${ }^{1}$
}

Résumé: Les caractères principaux de la géographie de Vidal de La Blache et de son école étaient connus au Japon jusque vers1940. Le mouvement de démocratisation du Japon après la deuxième guerre mondiale a mis la géographie française au premier plan pour reconstruire la géographie japonaise. En effet, la pensée géographique française était considérée comme un antidote à la géographie fasciste ou à la géopolitique. En ce qui concerne le redressement de la géographie japonaise d'après guerre, deux éléments ont influé pour la diriger vers les sciences sociales: d'une part, la tradition de la géographie vidalienne et, d'autre part, l'environnement scientifique, surtout marxiste. Même si les géographes japonais s'intéressaient beaucoup à la tradition française de la géographie régionale, ils ne l'ont jamais mise en pratique jusqu'à présent.

Mots-clés: Géographie japonaise, géographie française, marxisme, géographie régionale.

\begin{abstract}
RECEPTION AND TRANSFORMATION OF GEOGRAPHICAL IDEAS OF THE French GEOGRAPHY SCHOOL IN JAPAN - The main characteristics of the geography of Vidal de La Blache and of his school of thought were known in Japan until the early days of the 1940s, especially through the efforts made by a professor of the University of Tokyo, Koji lizuka. French geography was considered in Japan as representative of human geography in the world. It was used to counter Japanese geography, which was held to be environmentalistic and deterministic under the strong influence of German geography. After the Second World War, French geography offered an antithesis to fascist geography or to geopolitics during the movement of democratization in Japan, when geography was categorized as one of the disciplines of the social sciences. Two major elements played a role in the reconstruction of Japanese geography: the Vidalian tradition of French geography and the Marxist scientific environment. From the middle of the 1950s, Japanese geographers took an nterest in the methodologies of French regional geography, for example the important notion of way of life. Paradoxically, they never wrote original regional monographs of Japan.
\end{abstract}

Key-words: Japanese Geography, French geography, Marxism, regional geography.

\footnotetext{
1 Professeur de géographie. Faculté des Lettres, Université de Kyushu, 6-19-1 Hakozaki, Igashi-Ku, Fukuoka 812-81, Japon.

E-mail: nozawa@lit.kyushu-u.ac.jp
} 
Resumo: RECEPÇÃO E TRANSFORMAÇÃO DAS IDEIAS GEOGRÁFICAS DA ESCOLA FRANCESA NO JAPÃO - As principais características da Geografia de Vidal de La Blache e da sua escola de pensamento foram conhecidas no Japão no início dos anos de 1940, especialmente graças aos esforços do professor Koji lizuka, da Univeridade de Tóquio. O movimento de democratização do Japão depois da Segunda Guerra Mundial deu à geografia francesa o primeiro plano na reconstrução da geografia japonesa. Com efeito, a geografia francesa era considerada no Japão como representativa da geografia humana à escala mundial. No que diz respeito à renovação da geografia japonesa dois factos contribuíram para a orientar para as ciências sociais: por um lado a tradição geográfica vidaliana, por outro lado o meio científico marxista. A partir de meados dos anos de 1950 os geógrafos japoneses passaram a interessar-se pelas metodologias da geografia regional, por exemplo a importante noção de género de vida. Paradoxalmente nunca foram escritas monografias regionais do Japão.

Palavras-chave - Geografia japonesa, geografia francesa, marxismo, geografia regional.

Dans un article antérieur consacré à la réception de l'école française de géographie au Japon avant la deuxième guerre mondiale, je mentionnais les quelques observations suivantes (NOZAWA, 1980):

1) Les caractères principaux de la géographie de Vidal de La Blache et de son école étaient essentiellement connus au Japon jusque vers 1940 à travers les travaux de certains géographes et non géographes. Parmi eux, Koji lizuka a occupé une position centrale dans le processus d'introduction de la Géographie française dans l'archipel. Il a publié les traductions des Principes de géographie humaine de Paul Vidal de La Blache et de La Terre et l'évolution humaine de Lucien Febvre, respectivement en 1940 et en 1941, dans une collection de poche aux éditions Iwanami. Il en a profité pour bien situer Vidal de La Blache et son école dans l'histoire de la pensée géographique mondiale grâce aux introductions bibliographiques de ces traductions.

2) Avant la deuxième guerre mondiale, c'est la géographie physique qui prédominait au Japon par rapport à la géographie humaine. De plus, celle-ci était environnementaliste et déterministe. La plupart des géographes japonais considéraient la géographie française comme représentative de la géographie humaine dans le monde. C'est pourquoi on attendait de celle-ci qu'elle jouât le rôle d'antithèse face à une géographie japonaise jugée à la fois environnementaliste et déterministe sous l'influence de la géographie allemande, surtout ratzelienne.

3) Même si les vidaliens doivent leur renommée mondiale à leurs excellentes études ou monographies régionales, cette caractéristique de leur école n'a jamais été reconnue au Japon. Dans ce pays, les études régionales, plutôt locales, ont été effectuées par des professeurs des écoles normales dans chaque département, avec quelques bons résultats. Mais très peu de ces travaux ont été écrits par des universitaires et ceux-ci préféraient faire des études thématiques plutôt que régionales. Il faut toutefois noter que 
ces enquêtes sur des thèmes généraux étaient menées dans des régions particulières.

Si telle était la situation à la veille de la deuxième guerre mondiale, quelle a été ensuite le devenir des idées géographiques françaises au Japon?

\section{AUTOUR DE LA DÉFAITE DE LA DEUXIÈME GUERRE MONDIALE}

Cette époque est marquée d'une part, par l'effondrement de la géopolitique japonaise alors que la géographie japonaise entre dans le processus de démocratisation, et d'autre part, par l'intérêt porté à la pensée géographique française.

La géographie japonaise, comme je l'ai rappelé ci-dessus, s'est développée sous la très forte influence de la géographie allemande, et le Japon en a tiré ses idées géopolitiques. Celles-ci ont connu une vogue extraordinaire pendant la deuxième guerre mondiale à la lumière des idées japonaises sur les origines. Elles sont tombées en ruines en une seule nuit avec la défaite de 1945. Mais personne n'a encore vraiment entrepris jusqu'à présent au Japon d'études critiques conséquentes sur ce sujet, à une exception près, celle de Keiichi Takeuchi (TAKEUCHI, 1974; 1980; 1986). De jeunes géographes japonais commencent seulement à réexaminer ces idées géopolitiques. Il est clair, toutefois, que la géographie française n'a malheureusement pas été assez forte au Japon durant la guerre pour empêcher le développement de cette géopolitique.

Le mouvement de démocratisation du Japon après la deuxième guerre mondiale a mis la pensée géographique française au premier plan pour reconstruire la géographie japonaise. Celle-là a été considérée comme un antidote contre la géographie fasciste ou la géopolitique. Ce rôle d'antithèse n'était pas différent de la situation qui prévalait avant guerre: on regardait la géographie française comme une géographie humaine, possibiliste, tandis que la géographie allemande était perçue comme une géographie physique et déterministe.

L'éducation civique a connu un grand changement dans les enseignements primaires et secondaires au Japon après la défaite de 1945. On peut même dire qu'avant la guerre, elle n'existait pas. Elle n'a été créée réellement dans les enseignements primaires et secondaires qu'après la deuxième guerre mondiale. Or, la géographie au Japon a été située en tant que discipline dans le programme d'éducation civique.

Dans les universités, la géographie dite humaine se classait au sein des programmes de sciences humaines et sociales de l'enseignement général depuis la réforme universitaire d'après-guerre. On peut donc considérer qu'elle est devenue une discipline plutôt sociale. Cela revêt un sens important, car dans le Japon d'avant-guerre les sciences sociales, surtout marxistes, étaient littéralement brimées par l'Etat. Aussi, la conception de "la géographie comme science sociale" était bien vue, d'autant plus qu'elle restait ouverte au marxisme. Il ne fut alors question que de géographie française pour reconstruire la nouvelle géographie japonaise. C'est pourquoi Koji lizuka et ses disciples ont joué un rôle très important. Il n'est pas exagéré de dire que ses œuvres, basées sur la géographie vidalienne, ont servi de fondements pour la reconstruction de la géographie japonaise (IIZUKA, 1932, 1940, 1941, 1947, 1949, 1950). Et elles étaient par 
ailleurs très favorables à la pensée marxiste.

En France aussi, la géographie était en train d'évoluer vers les sciences sociales et subissait fortement l'influence de la pensée marxiste. C'était le cas de Pierre George qui, comme tout le monde le sait, a marqué ce courant de son influence.

Ainsi, on peut observer une même tendance dans les deux pays, comme illustrée notamment par Pierre George et ses disciples et par Koji lizuka et les siens. Je pense qu'il faut y voir une évolution parallèle plutôt qu'un rapport d'influence. Il est certain qu'il existait pas mal de traductions japonaises de Pierre George (celles des livres de format poche de la collection "Que sais-je?") et que ses travaux étaient assez bien considérés au Japon. Je ne pense pas, toutefois, qu'il y ait eu une influence directe de Pierre George sur l'orientation de la géographie japonaise vers une science sociale.

Toutes les disciplines sociales et humaines (même les sciences naturelles) subissaient alors l'influence de la pensée marxiste, surtout au Japon et peut-être en France aussi, après la deuxième guerre mondiale. N'y a-t-il pas des éléments dans la géographie vidalienne porteurs d'une orientation vers les sciences sociales ou le marxisme? Je pense que Koji lizuka, pour sa part, a trouvé cette direction dans la géographie de l'école française, celle de Vidal et de Demangeon. Quant à Pierre George, il a hérité de la géographie vidalienne, mais avec une position critique essayant de promouvoir une nouvelle géographie.

En ce qui concerne le redressement de la géographie japonaise d'après-guerre, nous pouvons donc souligner deux dimensions de l'influence qui l'a dirigée vers les sciences sociales: d'une part, la tradition de la géographie vidalienne et, d'autre part, l'environnement scientifique marqué par le marxisme.

\section{LES ÉTUDES JAPONAISES SUR LA GÉOGRAPHIE FRANÇAISE APRÈS LA DEUXIÈME GUERRE MONDIALE}

\section{1) Les études sur la notion de genre de vie:}

C'est d'abord par la notion de genre de vie que des géographes japonais ont commencé à aborder la géographie française dans les années 1950. Elle était une des notions les plus importantes de l'école française de géographie et a fait l'objet de nombreuses discussions entre les géographes français eux-mêmes (VIDAL DE LA BLACHE, 1911; GOTTMANN, 1947; SORRE, 1948; GILBERT, 1948; LE LANNOU, 1949; GEORGE, 1951; Derruau, 1969; Dumont, 1970). Au Japon, T. Takeo et Makoto Matsuda ont publié la même année leurs études sur la notion de genre de vie de l'école française de géographie, de Vidal de La Blache à Max. Sorre (TAKEO, 1955; MATSUDA, 1955). M. Matsuda a engagé des études plus détaillées sur l'évolution de la notion de genre de vie en France (MATSUDA, 1961). Mais K. Iizuka s'est interrogé sur l'efficacité de la notion de genre de vie, en posant la question "genre de vie ou mode de production?" (IIZUKA, 1950). Entre les deux notions, il préféra la dernière à la première. On trouve donc, ici, une preuve de l'influence de la pensée marxiste sur la nouvelle géographie japonaise inspirée des lectures de Pierre George. On sait que celui-ci avait déjà critiqué la notion de genre de vie dans des termes que l'on retrouve chez K. Iizuka (GEORGE, 1951). 
Toutefois, on notera que si Pierre George a privilégié l'importance des forces de production, $\mathrm{K}$. lizuka a accordé plus d'attention aux relations de production.

La notion de genre de vie des vidaliens fit une réapparition dans les années 1970 et 1980 chez les géographes humanistes des pays anglophones (sous l'influence de Anne Buttimer par exemple) au sein du mouvement critique contre la géographie positiviste, c'est-à-dire quantitative et théorique. Je me suis moi aussi engagé dans la critique de la substitution de la notion de genre de vie par celle de mode de production en me basant sur les œuvres de géographes humanistes (NOZAWA, 1983). Et j'en ai conclu que la notion de genre de vie était plus vaste et plus riche que celle de mode de production apportée par l'économie.

2) L'étude régionale dans la géographie française et ses méthodes:

L'étude régionale, comme tout le monde le sait, est une des principales caractéristiques de la géographie française. Cela a perduré, bien qu'on puisse constater de nombreux changements dans la démarche régionale. M. Matsuda a présenté l'évolution de la méthodologie des études régionales comme allant de la description picturale à la description structurale de la région dans l'école géographique française. Il est arrivé à la notion de "complexe géographique" en se plongeant dans la lecture des œuvres de l'école française de géographie (MATSUDA, 1962, 1967, 1971). J'ai également présenté une histoire des méthodes des études régionales françaises, à partir de quelques exemples d'études toutes récentes: celles de phénomènes psychologiques, sociologiques, culturels, et en plus celles s'inspirant la méthode de la géographie "chorématique" (NOZAWA, 1997).

Comme je l'ai déjà rappelé, les géographes humanistes anglo-saxons ont retrouvé les idées ou l'esprit de l'humanisme dans la géographie française vidalienne, lors du mouvement anti-positiviste des années 1970-1980. Toutefois, le regretté géographe japonais Takahiko Nishimura n'a pas trouvé ces éléments humanistes dans la géographie de Vidal lui-même, mais plutôt dans celle de ses disciples directs et indirects (c'est-à-dire un peu périphériques par rapport à l'école vidalienne): Jules Sion, Pierre Deffontaines, Pierre Gourou et Maurice Le Lannou (NISHIMURA, 1997).

Mais surtout, si les géographes japonais s'intéressaient beaucoup à la tradition française de la géographie régionale et s'ils en ont étudié les méthodes, ils ne les ont jamais mises en pratique jusqu'à présent pour traiter du Japon.

\section{LA PENSÉE GÉOGRAPHIQUE JAPONAISE DU POINT DE VUE FRANÇAIS}

En dernier lieu, je voudrais m'arrêter un instant sur un géographe français qui a fait de nombreuses études sur la pensée géographique japonaise et qui a ouvert de nouvelles voies pour l'épistémologie de la géographie humaine. Il s'agit d'Augustin Berque. Comme il faudrait trop de pages pour en traiter, je me contenterai ici d'aborder seulement un point essentiel de son travail.

A. Berque a lu et relu Fudo de Tetsuro Watsuji, un des chefs-d'œuvres du célèbre philosophe japonais. Celui-ci s'est démarqué de L'être et le temps de Martin Hei- 
degger. Watsuji y a remarqué et critiqué l'absence de la notion d'espace. Il s'est donc intéressé à traiter de l'importance de l'espace pour l'être humain. Selon Watstuji, le fudo, ou milieu, correspond à un moment structural de l'être humain et n'est pas pensé de façon environnementaliste et encore moins comme déterministe. C'est une notion phénoménologique et herméneutique. Pourtant, selon A. Berque, dans l'utilisation de cette notion pour interpréter les phénomènes de fudo, Watsuji tombe dans le déterminisme. A. Berque substitue donc une nouvelle notion, la médiance, au fudo pour donner un sens proche de l'herméneutique phénoménologique. La médiance est une trajection entre le sujet et l'objet, la nature et la culture, l'individu et le groupe. Il nous fait d'ailleurs remarquer une similitude entre la médiance et la notion de $b a$ (ou lieu) chez le grand philosophe japonais Kitaro Nishida. Quoi qu'il en soit, A. Berque réussit, par cette notion de médiance, à interpréter des aspects du fudo dans la vie quotidienne japonaise (BERQUE, 1982, 1986, 1990, 1993, 1996).

\section{EN GUISE DE CONCLUSION}

La géographie japonaise traverse aujourd'hui une crise à la fois institutionnelle et scientifique. Du côté institutionnel, les chaires de géographie des universités sont appelées à disparaître dans la réforme universitaire. Du côté scientifique, les nouveaux territoires exploités par des géographes étrangers, tels que les problèmes liés aux rapports entre la société et l'espace, les études culturelles, tous les phénomènes spatiaux, globaux ou locaux, etc., sont en voie d'être occupés par d'autres disciplines au Japon. Je pense que la géographie japonaise subit ici les conséquences d'avoir négligé l'étude des régions ou des lieux eux-mêmes. Elle est en passe de perdre ce qui pouvait la justifier comme projet scientifique. Or c'était précisément l'aspect le plus important de la géographie française, et celui que les géographes japonais n'ont malheureusement pas su s'approprier.

\section{BIBLIOGRAPHIE}

BERQue, A. (1982) - Vivre l'espace au Japon. P.U.F., Paris.

BERQUE, A. (1986) - Le sauvage et l'artifice - Les Japonais devant la nature. Gallimard, Paris.

BERQue, A. (1990) - Médiance. De milieux en paysages. Reclus, Montpellier-Paris.

BERQUE, A. (1993) - Du geste à la cité. Formes urbaines et lien social au Japon. Gallimard, Paris.

BERQue, A. (1996) - Etre humains sur la terre: Principes d'éthique de l'écoumène. Gallimard, Paris.

DerruaU, M. (1969) - Nouveau précis de géographie humaine. A. Colin, Paris.

DuMONT, F. (1970) - La dialectique de l'objet économique. Anthropos, Paris.

GEORGE, P. (1951) - Introduction à l'étude géographique de la population du monde. P.U.F. Paris.

GILBERT, A. (1948) - Les genres de vie dans le monde moderne. In Mélange géographique offert en hommage à M. Daniel Faucher: France méridionale et pays ibériques, Toulouse: 259- 
$-270$.

GotTMAnN, J. (1947) - De la méthode d'analyse en géographie humaine. Annales de Géographie, 56 (1), Paris: 1-12.

IIZUKA, K. (1932) - Shakai chirigaku nodoko (Orientation de la géographie sociale). Toko shoin, Tokyo.

IIZUKA, K. (1940) - Kaidai (Introduction bibliographique). In Principes de géographie humaine par Paul Vidal de La Blache (traduction japonaise). Iwanami bunko, Tokyo.

IIZUKA, K. (1941) - Kaidai (Introduction bibliographique). In La Terre et l'évolution humaine par Lucien Febvre (traduction japonaise). Iwanami bunko, Tokyo.

IIZUKA, K. (1947) - Chirigaku hihan - Shakai kagaku no ichi bumon toshiteno chirigaku (Critique de la géographie - La géographie comme une branche des sciences sociales), Teikoku shoin, Tokyo.

IIZUKA, K. (1949) - Jinbun chiri gakusetsushi - Hohoron notameno gakusetusiteki hansei (Histoire de la géographie humaine - Réflexion historique pour la méthodologie). Nihon hyoron sha, Tokyo.

IZZUKA, K (1950) - Jinbun chirigaku (Géographie humaine).Yuhi kaku,Tokyo.

LE LANNOU, M. (1949) - La géographie humaine. Flammarion, Paris.

MATSUDA, M. (1955) - Furansu jinbun chiri gakuhaniokeru seikatsu yoshiki gainen no hatten (L'évolution de la notion de genre de vie dans l'école française de géographie humaine). Kenkyu kiyo (Mie daigaku gakugei gakubu), 14, Tsu-Mie: 96-109.

MATSUdA, M. (1961) - Seikatsu yoshiki saiko (Some notes on "genre de vie" - modes of life). Jinbun chiri (Human geography), 13 (6), Kyoto:501-48.

MATSUDA, M. (1961) - Keikan to seikatsu yoshiki (Some notes on landscape - paysage - and modes of life - genre de vie). Jinbun chiri (Human geography), 17 (2), Kyoto: 113-133.

MATSUDA, M. (1962) - Chiiki no byoshateki kijutsukara kozoteki kijutsu he - Furansu gakuha no bai - (De la description picturale à la description structurale de la région - le cas de l'école française). Kenkyu kiyo (Mie daigakuk gakugei gakubu), 25, Tsu-Mie: 83-105.

MATSUDA, M. (1967) - Chirigaku niokeru kozo to kino (Structure et fonction dans la géographie). Osakgakugei daigaku kiyo, 15, Osaka: 179-189.

MATSUDA, M. (1971) - Chiriteki fukugotai gainen no tenkai (The concept of geographical complex, its development and effects). Jinbun chiri (Human geography), 23 (1), Kyoto: 74-90.

NISHIMURA, N. (1997) - Bunmei tokeikan (Civilisation et paysage). Chijin shobo, Kyoto.

NozAWA, H. (1980) - The French school of geography in Japan, 1926-1940. In Geographical languages in different times and places. Japanese contributions to the history of geographical thought, 3, Kyoto: 67-71. 
NozawA, H. (1983) - Seikatsu yoshiki omegurushomondai (Problèmes de la notion de genres de vie). Shien (Journal of History), 125, Fukuoka: 179-206.

NOZAWA, N. (1997) - Furansu chirigaku niokeru chiikikenkyuho (Méthodes de l'étude régionale dans la géographie française). In K. FuJIWARA (dir.). Chiiki kenkyuho (Méthodes de l'étude régionale). Asakura shoten, Tokyo:31-45.

SORRE, M. (1948) - La notion de genre de vie et sa valeur actuelle. Annales de Géographie, 57 (2-3), Paris: 97-108, 193-204.

TAKEO, T. (1955) - Furansu gakuha niokeru seikatsu yoshiki gainen (La notion de genre de vie dans l'école française). Ritsumeikan bungaku, 121 (1), Kyoto: 1-24.

TAKEUCHI, K. (1974) - Nhon niokeru geoporitiku to chirigaku (Geopolotics and geography in Japan). Hitotsubashi ronso (The Hitotsubashi Rewiew), 72 (2), Kunitachi-Tokyo: 169$-191$.

TAKEUCHI, K. (1980) - Geopolitics and geography in Japan: Re-examined. Hitotsubashi Journal of Social Studies, 12, Kunitachi-Tokyo: 14-24.

TAKEUCHI, K. (1986) - Geoporitiku no fukkatsu toseiji-chirigaku no atarashi tenkai - Geoporitiku saiko - (The Revival of geopolitics and the new trends in political geography). Hitotsubashi ronso (The Hitotsubashi Rewiew), 96 (5), Kunitachi-Tokyo: 523-546.

VIDAL DE LA Blache, P. (1911) - Les genres de vie dans la géographie humaine. Annales de Géographie, 20 (3-4), Paris: 193-212, 289-304. 